\title{
Anti-Apoptotic and Anti-0xidant Effects of Systemic Uridine Treatment in an Experimental Model of Sciatic Nerve Injury
}

\author{
Marzieh KARIMI KHEZRII ${ }^{1}$, Alper TURKKAN², Cansu KOC ${ }^{3}$, Berna SALMAN ${ }^{3}$, Pinar LEVENT ${ }^{3}$, Aysen CAKIR ${ }^{4}$, \\ Ilker Mustafa KAFA', Mehmet CANSEV ${ }^{3}$, Ahmet BEKAR ${ }^{1}$
}

\begin{abstract}
${ }^{1}$ Bursa Uludag University Faculty of Medicine, Department of Neurosurgery, Bursa, Turkey ${ }^{2}$ Medical Park Hospital, Neurosurgery Clinic, Bursa, Turkey

${ }^{3}$ Bursa Uludag University Faculty of Medicine, Department of Pharmacology, Bursa, Turkey

${ }^{4}$ Bursa Uludag University Faculty of Medicine, Department of Physiology, Bursa, Turkey

${ }^{5}$ Bursa Uludag University Faculty of Medicine, Department of Anatomy, Bursa, Turkey
\end{abstract}

Corresponding author: Ahmet BEKAR abekar@uludag.edu.tr

\section{ABSTRACT}

AIM: To investigate the anti-apoptotic and anti-oxidant effects of systemic uridine treatment in a rat model of sciatic nerve injury.

MATERIAL and METHODS: Thirty-two adult male rats were equally randomized to Sham, Control, U100, and U500 groups. Sham rats received a sham operation by exposing the right sciatic nerve without transection, while those in the Control, U100, and U500 groups underwent right sciatic nerve transection followed by immediate primary anostomosis. Sham and Control groups received saline $(0.9 \% \mathrm{NaCl})$ injections intraperitoneally (i.p.), while U100 and U500 groups received $100 \mathrm{mg} / \mathrm{kg}$ and $500 \mathrm{mg} / \mathrm{kg}$ uridine injections (i.p.), respectively, once a day for 7 days after the surgery. Rats in all the groups were sacrificed on the eighth day; sciatic nerve samples were analyzed for apoptosis by Western Blotting and for oxidation parameters including myeloperoxidase (MPO), malondialdehyde (MDA), superoxide dismutase (SOD), glutathione peroxidase (GPx) and catalase (CAT) by Enzyme-Linked Immunosorbent Assay (ELISA).

RESULTS: Uridine treatment at the dose of $500 \mathrm{mg} / \mathrm{kg}$ significantly decreased as apoptosis determined by Caspase-3/Actin ratio and exhibited significant anti-oxidant effects as determined by decreased levels of MPO and MDA as well as increased levels of SOD, GPx, and CAT compared to controls. Uridine at $100 \mathrm{mg} / \mathrm{kg}$ was only found to decrease the Caspase-3/Actin ratio, although it significantly decreased MDA and increased CAT levels compared to controls.

CONCLUSION: Treatment with uridine reduces apoptosis and oxidation in a rat model of sciatic nerve injury dose-dependently. Thus, uridine may be beneficial in peripheral nerve regeneration by exhibiting anti-apoptotic and anti-oxidant effects.

KEYWORDS: Peripheral nerve injury, Sciatic nerve, Uridine, Apoptosis, Anti-oxidant, Rat

ABBREVIATIONS: ANOVA: Analysis of variance, CAT: Catalase, CDP-choline: Cytidine-5'-diphosphocholine, DJ-1: Protein deglycase DJ-1, ELISA: Enzyme-Linked immunosorbent assay, GPx: Glutathione peroxidase, HIE: Hypoxic-ischemic encephalopathy, MDA: Malondialdehyde, MPO: Myeloperoxidase, PARK7: Parkinson disease protein 7, SDS-PAGE: Sodium dodecyl sulfate-polyacrylamide gel electrophoresis, SEM: Standard error of means, SOD: Superoxide dismutase, UDP: uridine-5'diphosphate, UTP: uridine-5'-triphosphate, WD: Wallerian degeneration

Marzieh KARIMI KHEZRI (1) : 0000-0002-6397-0966 Alper TURKKAN Cansu KOC
Berna SALMAN (1) : 0000-0001-8628-9578

Pinar LEVENT (D) : 0000-0001-9757-6716

Aysen CAKIR (1): 0000-0001-7729-7373
Ilker Mustafa KAFA (10): 0000-0001-8309-0934 Mehmet CANSEV (1) : 0000-0003-2918-5064

Ahmet BEKAR (10): 0000-0002-2716-1985 


\section{INTRODUCTION}

$\mathrm{P}$ eripheral nerve regeneration remains an unsolved problem in regenerative medicine due to several different factors including large amount of nerve tissue loss and emerging pathophysiological events. Although several approaches have been proposed to improve the recovery of sensory/motor function in peripheral nerve injury, no cure has yet been discovered to date. Currently, direct surgical treatment or nerve grafting is the most commonly practiced treatment of peripheral nerve injury; however, the clinical results obtained by using these methods are not fully satisfaction (18).

Newer approaches are required that need a comprehensive knowledge of the pathophysiology. Regarding the transection injury, the proximal segment of the neuron suffers degenerative alterations, whereas the distal segment undergoes Wallerian degeneration (WD) $(9,18)$. A broad range of events including apoptosis (29), oxidative stress (28), inflammation (27), degradation of the extracellular matrix (17), and many others can compromise the peripheral nerve injury. Therefore, strategies targeting such pathological processes may be beneficial to alleviate the consequences of peripheral nerve injuries.

Uridine is a major pyrimidine nucleoside in human blood circulation and breast milk $(5,33,35)$, and a precursor of membrane phospholipid synthesis through the pathway of Kennedy and Weiss (21). Uridine treatment has been shown to increase the levels of cytidine-5'-diphosphocholine (CDPcholine), a rate-limiting endogenous intermediate in membrane phospholipid synthesis, both in vitro (31), and in vivo (8). In addition to its metabolic roles, uridine has been shown to promote the axonal sprouting of pheochromocytoma-12 (PC12) cells in the culture (30), and exhibit neuroprotective properties in experimental models of central nervous system injuries $(1,6,13,14,22)$. In our previous study, we showed that uridine provided neuroprotection in newborn rat models of hypoxic-ischemic encephalopathy (HIE) $(6,22)$, and hyperoxic brain injury by inhibiting apoptotic cell death (14), and significantly reducing cognitive dysfunction in young adult rats during the long-term follow-up $(13,14)$. In addition, we recently reported the anti-oxidant activity of uridine in a newborn rat hyperoxic brain injury model (1).

Therefore, in the present study, we investigated the antiapoptotic and anti-oxidant effects of uridine treatment in rat sciatic nerve injury model with unilateral transection followed by primary anostomosis.

\section{MATERIAL and METHODS}

\section{Experimental Animals}

Thirty-two adult male Sprague-Dawley rats (220-350 g) were used in our study, which was conducted at the Bursa Uludag University Experimental Animal Research Center. Rats were purchased from this center after receiving the ethical approval from the Local Ethics Committee on Animal Use of Bursa Uludag University (Ratification number: 2017-15/04).

\section{Surgical Procedure}

All the rats were fastened up to the operation ground in the left lateral position under Sevoflurane (3\%) (Sevorane Liquid; Aesica Queenborough Ltd., Queenborough Kent, England) anesthesia. The surgical area was elevated with a $1-\mathrm{cm}$ thick rolled placed under the right thigh and inguinal region, and disinfected with $70 \%$ alcohol, followed by povidone iodine solution at $10 \%$ concentration (Bikar Inc., Istanbul, Turkey). A 3-cm skin incision in the posterolateral position was performed, extending from the right gluteal area to the posterior of the thigh, and the sciatic nerve was exposed by opening the surrounding fascia through a blunt dissection between the musculus gluteus superficialis and musculus biceps femoris (Figure 1A). Surgical interventions were performed using microsurgical methods under a surgical microscope (Zeiss Opmi-6; Carl Zeiss Meditec Inc. USA). The sciatic nerve was dissected and isolated from the surrounding tissues in the sciatic foramen to the point of the tibial and peroneal branches.

Besides the rats in the Sham group, a uniform incision was made using a metal dissector placed under the sciatic nerve and a full-thickness nerve transection was made $1 \mathrm{~cm}$ away from the sciatic foramen using a dermatome blade (Figure 1B). Proximal and distal nerve stumps were then sutured microsurgically using 8-0 polypropylene sutures (Prolene, Ethicon Ltd., Somerville NJ, USA) at $180^{\circ}$ aperture, and a primary anastomosis was provided (Figure 1C). Maximum care was taken in order not to creat tension at the anastomosis. After completing the procedure, the skin and fascia were closed.

\section{Treatments}

The rats were randomized to the Sham, Control, U100, and U500 groups, containing 8 rats in each group. Sham rats received a sham operation by exposing the right sciatic nerve without transection, while those in the Control, U100, and U500 groups underwent right sciatic nerve transection followed by immediate primary anostomosis. Sham and Control groups were injected with $1 \mathrm{~mL} / \mathrm{kg}$ serum saline $(0.9 \% \mathrm{NaCl})$ through the intraperitoneal (i.p.) route, while U100 and U500 groups received $100 \mathrm{mg} / \mathrm{kg}$ and $500 \mathrm{mg} / \mathrm{kg}$ uridine (i.p.; dissolved in saline), respectively, once a day for 7 days immediately after the surgery.

All the rats were returned to their cages (4 rats in each cage) and recovered spontaneously from anesthesia with free access to food and water under a 12-h light/dark cycle.

\section{Nerve Tissue Sampling}

Rats in all the groups were sacrificed under anesthesia on the eighth day of the surgery and sciatic nerve samples were obtained from the Sham, Saline, U100, and U500 groups. After opening the incision, right sciatic nerves were resected en bloc by including the sectioned and sutured portion of the nerve. Specimens were frozen at $-20^{\circ} \mathrm{C}$ for further analyses of molecular biological and biochemical parameters.

\section{Apoptosis Evaluation}

The effect of uridine treatment on apoptotic cell death following 


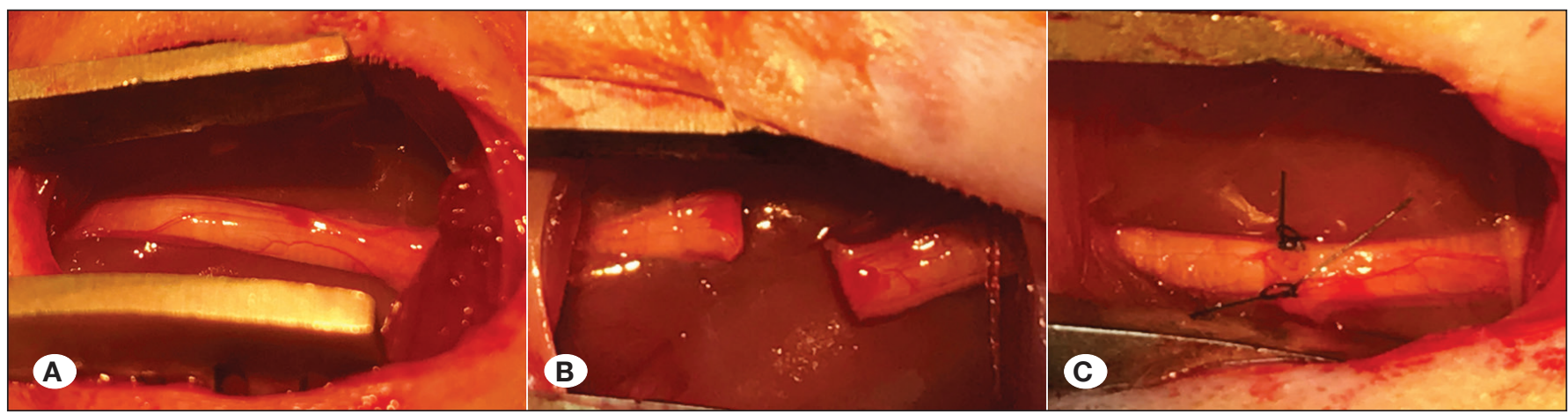

Figure 1: Representative images of the operational procedure: A) the sciatic nerve was exposed by opening the surrounding fascia by a blunt dissection between musculus gluteus superficialis and musculus biceps femoris, B) a full-thickness nerve transection was made $1 \mathrm{~cm}$ away from the sciatic foramen using a dermatome blade, C) proximal and distal nerve stumps were then sutured microsurgically with 8-0 polypropylene sutures at $180^{\circ}$ aperture, and primary anastomosis was provided.

sciatic nerve injury was evaluated by analyzing Caspase-3 protein expression using the Western Blotting method. Sciatic nerve samples were homogenized in deionized water and the total protein contents of the homogenates were determined according to the Lowry method (25). The homogenates were then boiled for 5 minutes in Laemmli buffer (23), and loaded onto sodium dodecyl sulfate-polyacrylamide gel electrophoresis (SDS-PAGE; Mini Protean IIII, Bio-Rad, Hercules, CA, USA) at volumes containing equal amounts of protein. The proteins were separated by SDS-PAGE and transferred onto polyvinylidene fluoride (PVDF) membranes (Millipore, Billerica, MA, USA). The membranes were blocked with $5 \%$ milk powder solution and incubated overnight with the specific anti-Caspase-3 primary antibody (1: 1000, Cell Signaling Technology, Danvers, MA, USA). Next day, the membranes were incubated with the appropriate secondary antibody (1: 5000, Cell Signaling Technology, Danvers, MA, USA), followed by incubation for 1 hour with chemoluminescence detection reagent using a commercially-available kit (Millipore, Billerica, MA, USA). Protein bands were digitized and optical densities of Caspase-3 protein were analyzed using a digital scanner (CDigit, LI-COR Biotechnology, Lincoln, NE, USA). The membranes were then washed with a stripping buffer (Thermo Fisher Scientific, Rockford IL, USA) and re-treated with an anti-Actin primary antibody (Millipore, Temecula, CA, USA) to analyze Actin levels using the same procedure. Levels of Caspase-3 proteins were presented in comparison to Actin, and the results were presented as percent changes compared to the Sham group.

\section{Biochemical Evaluation}

In order to investigate the effect of uridine treatment on oxidant and anti-oxidant parameters, levels of myeloperoxidase (MPO), malondialdehyde (MDA), glutathione peroxidase (GPx), superoxide dismutase (SOD), and catalase (CAT) were measured in nerve tissue homogenates using commercial ELISA kits (YL Biotech, Shangai, PRC). Measurements were performed spectrophotometrically (mQuant, Biotek, Winooski, VT) by adhering to kit procedures.

\section{Statistical Analyses}

Statistical analyses were performed using Sigma Plot version 12.0 software. Data were presented as mean \pm standard error of means (SEM). Comparisons were made using One-Way Analysis of Variance (ANOVA) followed by the post hoc Tukey test. A $p$ value less than 0.05 was considered to be statistically significant.

\section{RESULTS}

\section{Effects of Uridine Treatment on Apoptosis}

Sciatic nerve injury resulted in significantly $(p<0.001)$ enhanced levels of the apoptotic marker Caspase-3 in the Control group compared to the Sham group. Uridine treatment at $100 \mathrm{mg} /$ kg (U100 group) was found to decrease Caspase-3 levels, although the decrease was not statistically significant. On the other hand, treatment with $500 \mathrm{mg} / \mathrm{kg}$ uridine (U500 group) significantly $(p<0.05)$ decreased Caspase-3 levels compared to the Control group (Figure 2).

\section{Effects of Uridine Treatment on Oxidation Parameters}

Levels of the oxidant enzymes myeloperoxidase (MPO) and malondialdehyde (MDA) were significantly increased $(p<0.001)$ following sciatic nerve injury in the Control group compared to the Sham group (Table I). Treatment with $100 \mathrm{mg} / \mathrm{kg}$ uridine (U100 group) significantly decreased MDA levels $(p<0.05)$, while it was found to only decrease MPO levels. On the other hand, levels of both MPO and MDA were significantly decreased $(p<0.001)$ in the U500 group compared to the Control group.

Levels of the anti-oxidant enzymes superoxide dismutase (SOD), glutathione peroxidase (GPx), and catalase (CAT) were significantly decreased $(p<0.001)$ following sciatic nerve injury in the Control group compared to the Sham group (Table I). Treatment with $100 \mathrm{mg} / \mathrm{kg}$ uridine (U100 group) significantly increased CAT levels $(p<0.05)$, while it was found to only increase SOD and GPx levels. On the other hand, levels of SOD $(p<0.001)$, GPx $(p<0.01)$, and CAT $(p<0.001)$ were all increased significantly in the U500 group compared to the Control group. 
Karimi Khezri M. et al: Effects of Uridine on Sciatic Nerve Injury

Table I: Effects of Uridine Treatment on Oxidation Parameters in Sciatic Nerve Injury

\begin{tabular}{|c|c|c|c|c|}
\hline & Sham & Control & U100 & U500 \\
\hline MDA (nmol/ml) & $0.68 \pm 0.02^{\star \star \star}, \# \#$ & $0.93 \pm 0.03$ & $0.82 \pm 0.02^{*}$ & $0.72 \pm 0.01^{* \star *, \#}$ \\
\hline SOD (ng/ml) & $1.85 \pm 0.03^{\star \star \star \star \# \# ~}$ & $1.45 \pm 0.04$ & $1.60 \pm 0.04$ & $1.78 \pm 0.04^{\star \star \star * \#}$ \\
\hline GPx (ng/ml) & $14.6 \pm 0.3^{\star \star \star, \# \#}$ & $11.5 \pm 0.1$ & $12.6 \pm 0.5$ & $13.4 \pm 0.2^{\star \star}$ \\
\hline
\end{tabular}

Levels of Myeloperoxidase (MPO), Malondialdehyde (MDA) Superoxide Dismutase (SOD), Glutathione Peroxidase (GPx) and Catalase (CAT) were analyzed by ELISA method using commercially-available kits. ${ }^{*} p<0.05,{ }^{* *} p<0.01$ and ${ }^{* * *} p<0.001$ compared to Control group; $\# p<0.05$ and $\# p<0.01$ compared to $U 100$ group.

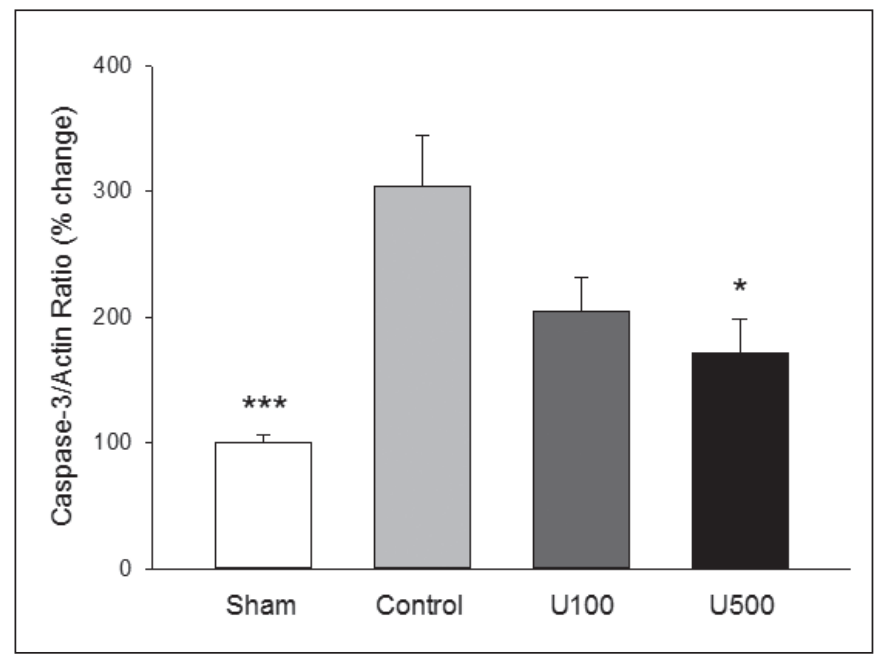

Figure 2: Effects of uridine treatment on apoptosis in sciatic nerve injury. Data were obtained by Western Blotting and presented as the percentage change in Caspase-3/Actin ratio. ${ }^{*} p<0.05$ and ${ }^{\star \star *} \mathrm{p}<0.001$ compared to the Control group.

\section{DISCUSSION}

For the first time, our data show that systemic uridine treatment dose-dependently exhibits anti-apoptotic and anti-oxidant effects in a peripheral nerve injury rat model, performed by full-thickness transection, followed by primary anostomosis of the rat sciatic nerve.

Peripheral nerve injury causes several physiopathological changes in both the distal and proximal regions of the nerve. In a nerve that survives after injury, the proximal part of the nerve is usually degenerated by the action of calcium flow as well as calcium-associated protease activation up to the first Ranvier node $(12,34)$. The proximal parts swell under the influence of proteins in the cytoskeleton and cellular organelles that reach the target by axoplasmic transfer (34). Damaged axon requires that the growth cone is dynamically-prolonged $(11,34,37)$. Synthesis of local proteins stimulates the initial changes without requiring the participation of transcriptional alterations (34). The pre-existing elements of the cytoskeleton in axons are transferred from the proximal nerve stump to the regenerated axons (34). Microtubules function as bridges that carry the materials necessary for elongation between existing and newly-forming axons (34). Regenerated axon portions being $t$ elongate from the initial Ranvier node at lesion's proximal site (34). The fine nerve fibers extend from the supporting microenvironment of the proximal nerve shoots to the distal nerve end $(11,34,37)$. Extracellular matrix proteins, cell adhesion molecules, and neurotrophic factors can be classified either as a direct or an indirect contributing factor to the regeneration of axons $(11,34,37)$.

Axon-to-axon anastomosis is still widely accepted as the gold-standard (15) treatment for peripheral nerve injury, while factors such as the quality of axonal growth, number of viable neurons, orientation of the regenerated axon, and the condition of the target tissue are very important in ideal nerve repair $(19,26,32)$. In addition, a number of pathophysiological events including apoptosis (29), oxidative stress (28), inflammation (27), degradation of the extracellular matrix (17), and many others may compromise the recovery process in peripheral nerve injury. To date, several surgical and nonsurgical therapeutic approaches have been proposed for the anatomical and functional recovery of injured peripheral nerves (18).

Surgical approaches including direct nerve repair, nerve grafting, nerve transfer, fibrin glue, nerve conduits, and cellbased therapy do not offer complete recovery. They are generally time-consuming and expensive, and have the risk of donor unavailability and immunosuppression (18). With regard to the non-surgical approaches, available medications such as analgesics, opioids, and corticosteroids that serve as the firstline options for treatment are only helpful in providing relief from pain. Nevertheless, these medications cannot accelerate the regeneration process and are not useful in providing functional recovery (18). Alternative therapeutic approaches with phytochemicals including quercetin, ursocolic acid, curcumin, and several others have been proposed to confer benefit with experimental evidence, while most of them cause side effects at human doses (18). Therefore, newer approaches are required for peripheral nerve injury treatment having governing optimum properties for a medication, such as causing ignorable side effects.

In the present study, we investigated the effects of uridine, an endogenous pyrimidine nucleoside $(5,33,35)$, in a peripheral 
nerve injury rat model by performing full-thickness transection and immediate end-to-end anostomosis of the sciatic nerve. Uridine was tested in peripheral nerve injury for the following reasons: (i) uridine is a precursor of CDP-choline $(8,31)$ which improved regeneration of the nerve as well as functional recovery in the sciatic nerve injury models $(4,16,20)$, (ii) uridine promoted nerve proliferation in cultured PC12 cells resulting in axonal growth and sprouting (30), (iii) uridine inhibited inflammation and fibrosis in bleomycin-induced lung injury, (10) and (iv) uridine provided neuroprotection in models of hypoxic-ischemic and hyperoxic brain damage $(1,6,13,14,22)$.

Herein, we show here for the first time in peripheral nerve tissue that systemic uridine administration at the dose $(500 \mathrm{mg} / \mathrm{kg})$ shown previously to provide neuroprotection $(1,6,13,14,22)$, reduces Caspase- 3 levels and alleviates oxidation, behaving as an anti-apoptotic and anti-oxidant agent.

Our data are in agreement with previous findings of experimental brain damage models. We previously showed that uridine exhibited neuroprotective activity by suppressing apoptotic cell death in neonatal rat $\operatorname{HIE}(6,22)$ and significantly reduced cognitive dysfunction in young adult rats during long-term follow-up (13). The anti-apoptotic activity of uridine may depend on its precursor function to produce uridine5'-diphosphate (UDP) and uridine-5'-triphosphate (UTP) (8), which activates P2Y receptors (7) that inhibit apoptosis (2).

In addition, treatment with uridine alleviated hyperoxic brain injury in newborn rats (14) and we recently showed that this effect is mediated by reducing oxidation caused by hyperoxia (1). The finding that uridine behaves as an anti-oxidant may be associated with its function in restoring DJ-1 levels following oxidative stress in nerve tissue (1). DJ-1 (Protein deglycase DJ-1, also known as Parkinson disease protein 7 [PARK7]) (3) functions as a redox-sensitive chaperone to protect tissues against oxidative stress and cell death (24). Hence, in cases where DJ-1 levels are decreased, oxidative stress is triggered, which in turn contributes to cell death, as shown in a neuronal cell line (36) or in the immature brain following hyperoxic insult (3). Our recent data showed that uridine enhanced the decreased levels of $\mathrm{DJ}-1$ in newborn rats subjected to hyperoxic brain injury, suggesting a mechanism by which uridine may act as an anti-oxidant.

The effects of uridine in the present sciatic nerve injury model on apoptosis and oxidation were dose-dependent. We chose an effective dose $(500 \mathrm{mg} / \mathrm{kg})$ and a non-effective dose (100 mg/kg) from our previous studies $(1,6,13,14,22)$, which provided and did not provide neuroprotection, respectively, in newborn rat models of HIE and hyperoxic brain injury. We observed that $100 \mathrm{mg} / \mathrm{kg}$ uridine treatment only decreased MDA levels and increased CAT levels significantly, but did not reduce apoptosis. On the other hand, uridine at 500 $\mathrm{mg} / \mathrm{kg}$ dose significantly decreased Caspase-3, MPO and MDA levels, while significantly increasing SOD, GPX, and CAT levels. Therefore, in good agreement with our previous findings $(1,6,13,14,22)$, uridine dose-dependently provided anti-apoptotic and anti-oxidant effects.

\section{CONCLUSION}

In conclusion, we showed for the first time that treatment with systemic uridine exhibits anti-apoptotic and anti-oxidant effects in a peripheral nerve injury rat model performed by full-thickness transection followed by primary anostomosis of the rat sciatic nerve. The effects of uridine in this model were dose-dependent and comparable with previous reports of brain injury models. Hence, uridine may confer a therapeutic benefit in the treatment of peripheral nerve injury.

\section{ACKNOWLEDGEMENTS}

The present study has been supported by Bursa Uludag University Scientific Research Projects Commission (Approval number: OUAP(T)-2018/9).

\section{REFERENCES}

1. Al N, Cakir A, Sevinc C, Cansev M, Alkan T: Antioxidative effects of uridine in a neonatal rat model of hyperoxic brain injury. Turk J Med Sci 50(8):2059-2066, 2020

2. Arthur DB, Georgi S, Akassoglou K, Insel PA: Inhibition of apoptosis by P2Y2 receptor activation: Novel pathways for neuronal survival. J Neurosci 26:3798-3804, 2006

3. Bendix I, Weichelt U, Strasser K, Serdar M, Endesfelder S, von Haefen C, Heumann R, Ehrkamp A, Felderhoff-Mueser U, Sifringer M: Hyperoxia changes the balance of the thioredoxin/ peroxiredoxin system in the neonatal rat brain. Brain Res 1484:68-75, 2012

4. Caner B, Kafa MI, Bekar A, Kurt MA, Karli N, Cansev M, Ulus IH: Intraperitoneal administration of CDP-choline or a combination of cytidine plus choline improves nerve regeneration and functional recovery in a rat model of sciatic nerve injury. Neurol Res 34:238-245, 2012

5. Cansev M: Uridine and cytidine in the brain: Their transport and utilization. Brain Res Rev 52:389-397, 2006

6. Cansev M, Minbay Z, Goren B, Yaylagul EO, Cetinkaya M, Koksal N, Alkan T: Neuroprotective effects of uridine in a rat model of neonatal hypoxic-ischemic encephalopathy Neurosci Lett 542:65-70, 2013

7. Cansev M, Orhan F, Yaylagul EO, Isik E, Turkyilmaz M, Aydin S, Gumus A, Sevinc C, Coskun N, Ulus $\mathrm{IH}$, Wurtman RJ: Evidence for the existence of pyrimidinergic transmission in rat brain. Neuropharmacology 91:77-86, 2015

8. Cansev M, Watkins CJ, Van der Beek EM, Wurtman RJ: Oral uridine-5'-monophosphate (UMP) increases brain CDPcholine levels in gerbils. Brain Res 1058:101-108, 2005

9. Carroll SL, Worley SH: Wallerian degeneration. In: Stein JP (ed), Reference Module in Neuroscience and Biobehavioral Psychology. Amsterdam, Netherlands: Elsevier, 2017

10. Cicko S, Grimm M, Ayata K, Beckert J, Meyer A, Hossfeld M, Zissel G, Idzko M, Müller T: Uridine supplementation exerts anti-inflammatory and anti-fibrotic effects in an animal model of pulmonary fibrosis. Respir Res 16:105, 2015

11. Fu SY, Gordon T: The cellular and molecular basis of peripheral nerve regeneration. Mol Neurobiol 14:67-116, 1997 
12. George EB, Glass JD, Griffin JW: Axotomy-induced axonal degeneration is mediated by calcium influx through ionspecific channels. J Neurosci 15:6445-6452, 1995

13. Goren B, Cakir A, Ocalan B, Serter Kocoglu S, Alkan T, Cansev $\mathrm{M}$, Kahveci N: Long-term cognitive effects of uridine treatment in a neonatal rat model of hypoxic-ischemic encephalopathy. Brain Res 1659:81-87, 2017

14. Goren B, Cakir A, Sevinc C, Serter Kocoglu S, Ocalan B, Oy C, Minbay Z, Kahveci N, Alkan T, Cansev M: Uridine treatment protects against neonatal brain damage and long-term cognitive deficits caused by hyperoxia. Brain Res 1676:57-68, 2017

15. Griffin JW, Hogan MV, Chhabra AB, Deal DN: Peripheral nerve repair and reconstruction. J Bone Joint Surg Am 95:21442151, 2013

16. Gundogdu EB, Bekar A, Turkyilmaz M, Gumus A, Kafa IM, Cansev M: CDP-choline modulates matrix metalloproteinases in rat sciatic injury. J Surg Res 200:655-663, 2016

17. Hughes PM, Wells GM, Clements JM, Gearing AJ, Redford EJ, Davies M, Smith KJ, Hughes RA, Brown MC, Miller KM: Matrix metalloproteinase expression during experimental autoimmune neuritis. Brain 121:481-494, 1998

18. Hussain G, Wang J, Rasul A, Anwar H, Qasim M, Zafar S, Aziz N, Razzaq A, Hussain R, de Aguilar JG, Sun T: Current status of therapeutic approaches against peripheral nerve injuries: A detailed story from injury to recovery. Int J Biol Sci 16:116134, 2020

19. Kanaya F, Firrell JC, Breidenbach WC: Sciatic function index, nerve conduction tests, muscle contraction, and axon morphometry as indicators of regeneration. Plast Reconstr Surg 98:1264-1271, 1996

20. Kaplan T, Kafa IM, Cansev M, Bekar A, Karli N, Taskapilioglu $\mathrm{MO}$, Kanar F: Investigation of the dose-dependency of citicoline effects on nerve regeneration and functional recovery in a rat model of sciatic nerve injury. Turk Neurosurg 24:54-62, 2014

21. Kennedy EP, Weiss SB: The function of cytidine coenzymes in the biosynthesis of phospholipides. J Biol Chem 222:193214, 1956

22. Koyuncuoglu T, Turkyilmaz M, Goren B, Cetinkaya M, Cansev $\mathrm{M}$, Alkan T: Uridine protects against hypoxic-ischemic brain injury by reducing histone deacetylase activity in neonatal rats. Restor Neurol Neurosci 33:777-784, 2015

23. Laemmli UK: Cleavage of structural proteins during the assembly of the head of bacteriophage T4. Nature 227:680685,1970
24. Lev N, Ickowicz D, Melamed E, Offen D: Oxidative insults induce DJ-1 upregulation and redistribution: Implications for neuroprotection. Neurotoxicology 29:397-405, 2008

25. Lowry OH, Rosebrough NJ, Farr AL: Protein measurement with the Folin phenol reagent. J Biol Chem 193:265-275, 1951

26. Lundborg G: A25-year perspective of peripheral nerve surgery: Evolving neuroscientific concepts and clinical significance. J Hand Surg Am 25:391-414, 2000

27. Nadeau S, Filali M, Zhang J, Kerr BJ, Rivest S, Soulet D, Iwakura Y, de Rivero Vaccari JP, Keane RW, Lacroix S: Functional recovery after peripheral nerve injury is dependent on the pro-inflammatory cytokines IL-1 $\beta$ and TNF: Implications for neuropathic pain. J Neurosci 31:12533-12542, 2011

28. Naik AK, Tandan SK, Dudhgaonkar SP, Jadhav SH, Kataria M, Prakash VR, Kumar D: Role of oxidative stress in pathophysiology of peripheral neuropathy and modulation by $\mathrm{N}$-acetyl-L-cysteine in rats. Eur J Pain 10:573-579, 2006

29. Oliveira ALR: Apoptosis of sensory neurons and satellite cells after sciatic nerve transection in C57BL/6J mice. Braz J Med Biol Res 34:375-380, 2001

30. Pooler AM, Guez DH, Benedictus R, Wurtman RJ: Uridine enhances neurite outgrowth in nerve growth factordifferentiated pheochromocytoma cells. Neuroscience 134:207-214, 2005

31. Richardson UI, Watkins CJ, Pierre C, Ulus IH, Wurtman RJ: Stimulation of CDP-choline synthesis by uridine or cytidine in PC12 rat pheochromocytoma cells. Brain Res 971:161-167, 2003

32. Terzis JK, Sun DD, Thanos PK: Historical and basic science review: Past, present, and future of nerve repair. J Reconstr Microsurg 13:215-225, 1997

33. Thorell L, Sjöberg LB, Hernell O: Nucleotides in human milk: Sources and metabolism by the newborn infant. Pediatr Res 40:845-852, 1996

34. Turan SK, Onur MA, Demiralp FDO: Investigation of axonal regeneration of Triturus ivanbureschi by using physiological and proteomic strategies. J Biosci 44:145, 2019

35. Wurtman RJ, Regan M, Ulus I, Yu L: Effect of oral CDP-choline on plasma choline and uridine levels in humans. Biochem Pharmacol 60:989-992, 2000

36. Yokota T, Sugawara K, Ito K, Takahashi R, Ariga H, Mizusawa $\mathrm{H}$ : Down regulation of $\mathrm{DJ}-1$ enhances cell death by oxidative stress, ER stress, and proteasome inhibition. Biochem Biophys Res Commun 312:1342-1348, 2003

37. Zochodne DW: Neurobiology of Peripheral Nerve Regeneration. London: Cambridge University Press, 2008 